\title{
Scenarios to Manage the Demand for N95 Respirators for Healthcare Workers During the COVID-19 Pandemic
}

This article was published in the following Dove Press journal: Risk Management and Healthcare Policy

\author{
Yinxiaohe Sun' \\ Hirono Otomaru ${ }^{1,2 *}$ \\ Sharon Esi Duoduwa Quaye ${ }^{\prime}$ \\ Jyoti Somani ${ }^{3}$ \\ Natasha Bagdasarian ${ }^{3}$ \\ Darius LL Beh ${ }^{3}$ \\ Dale A Fisher (iD ${ }^{3,4}$ \\ Alex R Cook' \\ Borame L Dickens ${ }^{\prime}, *$ \\ 'Saw Swee Hock School of Public Health, \\ National University of Singapore and \\ National University Health System, \\ Singapore; ${ }^{2}$ Department of Virology, \\ Tohoku University Graduate School of \\ Medicine, Sendai, Japan; ${ }^{3}$ Division of \\ Infectious Diseases, National University \\ Hospital, Singapore; ${ }^{4}$ Yong Loo Lin \\ School of Medicine, National University \\ of Singapore, Singapore, Singapore
}

*These authors contributed equally to this work

Correspondence: Hirono Otomaru Email otomaruh@med.tohoku.ac.jp
Background: By estimating N95 respirator demand based on simulated epidemics, we aim to assist planning efforts requiring estimations of respirator demand for the healthcare system to continue operating safely in the coming months.

Methods: We assess respiratory needs over the course of mild, moderate and severe epidemic scenarios within Singapore as a case study using a transmission dynamic model. The number of respirators required within the respiratory isolation wards and intensive care units was estimated over the course of the epidemic. We also considered single-use, extended-use and prolonged-use strategies for N95 respirators for use by healthcare workers treating suspected but negative (misclassified) or confirmed COVID-19 patients.

Results: Depending on the confirmed to misclassified case ratio, from 1:0 to 1:10, a range of 117.1 million to 1.1 billion masks are required for single-use. This decreases to 71.6-784.4 million for extended-use and 12.8-148.2 million for prolonged-use, representing a $31.8-38.9 \%$ and $86.5-89.1 \%$ reduction, respectively.

Conclusion: An extended-use policy should be considered when short-term supply chains are strained but planning measures are in place to ensure long-term availability. With severe shortage expectations from a severe epidemic, as some European countries have experienced, prolonged use is necessary to prolong supply.

Keywords: COVID-19 pandemic, mathematical modelling, policy, healthcare resources, N95 respirators

\section{Introduction}

The COVID-19 pandemic is posing severe challenges to healthcare systems, leading to shortages of personal protective equipment (PPE) which leaves frontline healthcare workers $(\mathrm{HCW})$ at grave risk. Among the lessons learned during the outbreaks of Severe Acute Respiratory Syndrome coronavirus (SARS-CoV) in 2003 and the Middle East Respiratory Syndrome coronavirus (MERS-CoV) in 2012 were the need for stringent infection control in healthcare settings, clear criteria for isolation and quarantine measures, and continued evaluation of the effectiveness of PPE in infection prevention. ${ }^{1,2}$

The World Health Organisation (WHO) has warned of the risk of the global supply of PPE rapidly depleting. ${ }^{3}$ Despite these efforts, hospitals have faced severe shortages of PPE. ${ }^{4}$ Shortages of PPE lead to healthcare workers being dangerously ill-prepared to care for patients with COVID-19. ${ }^{5}$ Studies showed that N95 respirators (N95s) offer good protection against viral respiratory pathogens, especially 
during aerosol-generating procedures or when a patient's COVID-19 status is positive or unknown. ${ }^{6-8}$ N95s are of critical importance for confronting the SARS-CoV-2 pandemic among the various PPEs. Strategies to optimise the usage of N95s are therefore vital. These should focus on conserving N95 use while providing adequate protection for HCWs working with COVID-19 patients. HCWs may need to have contact with confirmed or suspected patients and their surroundings within inpatient facilities, often over an entire work shift. Extended-use-or even reuseof N95s has therefore been suggested, with recommendations in place to use respirators for up to 4 hours if N95s are in short supply. ${ }^{9-11}$ A study that screened 27 countries or regions revealed that only 5 countries (19\%) allowed extended use; 2 countries (7\%) mentioned reuse; and 3 countries (11\%) recommended both strategies for rationing N95 respirators. ${ }^{12}$ Several ways to decontaminate N95s for reuse have also been well studied. ${ }^{13}$

The potential extended-use of N95s requires serious consideration as the demand for respirators will increase in response to rising case counts, especially among those with severe disease who require intensive care and prolonged hospitalization. One in five confirmed cases in a hospital setting in China were reported to have severe symptoms requiring long stays at hospital facilities, ${ }^{14}$ while a quarter of those required admission to the intensive care unit (ICU) due to complications of acute respiratory distress syndrome, arrhythmia and shock, which significantly increase hospital stay. ${ }^{15}$ The same study observed a median hospital stay of 10 days (IQR 7-14) among those discharged. Per patient, multiple N95s are consequently required to avoid transmission between $\mathrm{HCWs}$ and patients.

In Singapore, a city of $\sim 5.6 \mathrm{M}$ people in the Malay Archipelago, 51,197 cases have been reported as of 2020-07-28. ${ }^{16}$ This number is expected to rise in the coming months, making it paramount to project the number of N95s required to ensure adequate provisioning. Here we estimate the number of N95s required over the course of the epidemic under three strategies: single-use, extended-use and prolonged-use. Through estimating the demand, we aim to aid planning efforts by assessing what preparations are required over the coming months for the healthcare system to run with the maximized possible protection for its workers. Although described for the Singapore setting, our results hold for planners in similarly sized cities elsewhere.

\section{Methods}

We developed a Susceptible-Exposed-Infectious-Recovered (SEIR) model to estimate the number of cases with COVID-
19 being present in healthcare facilities, and necessary N95 use under three policies: single-use, extended-use and prolonged-use. For planning purposes, we developed three variants of the SEIR model which describe the relative outbreak size, labelled as mild, severe and moderate.

\section{Epidemic Scenarios}

In the SEIR model, we classified the outcomes of COVID19 infection into six levels, relating to the need for hospitalization or being admitted to the ICU, and the risk of onward transmission (Figure 1). These were: level 1 for asymptomatic infections; level $2 \mathrm{a}$ for mild symptoms that remain undiagnosed at healthcare facilities; level $2 b$ for mild symptomatic ambulatory cases which are identified; level 3 for those who required hospitalisation but not the ICU for their recovery; level 4 for those who entered ICU on admission; and level 5 for those dying among levels 3 and 4 . We combined levels $3-5$ as severe and levels $1-2$ as mild. We also combined levels $2 \mathrm{~b}-4$ as known and levels $1-2 \mathrm{a}$ as cryptic i.e. cases which are not documented by the healthcare system.

We varied the parameters in the mild to severe scenarios regarding the: (i) proportion of cases seeking medical attention (30\%, 50\% and 70\% separately), (ii) proportion of cases requiring hospitalisation $(10 \%, 20 \%$ and $30 \%$, respectively), (iii) proportion of cases admitted in ICU among hospitalised cases $(20 \%, 30 \%$ and $40 \%$ from mild scenarios to severe scenarios), (iv) case fatality rate $(4.2 \%$, $1.8 \%$ and $0.43 \%),{ }^{17}$ and (v) the basic reproduction number (2.0, 1.5, and 1.5) ${ }^{18}$ (Supplementary Figure 1, Supplementary Table 1). Full details of the methods are presented in the Supplementary Information.

All analyses were performed by $\mathrm{R}$ version 3.6.2. A web application was developed in R Shiny under the link: https://sshsphdemos.shinyapps.io/COVID2019_v4b/.

\section{N95 Respirators Policies}

Three N95 use policy scenarios were suggested based on the frequency of these inpatient visits (Supplementary Figures 2 and 3 ): single-use, extended-use and prolongeduse. Under the single-use policy scenario, new N95s are used by all staff for each patient encounter. For the extended-use policy scenario, new N95s are used after every three patient encounters for nurses, while other HCWs don a fresh set for each encounter. For prolongeduse, all staff change N95s after seeing 10 ICU patients concurrently and/or after every four hours. 


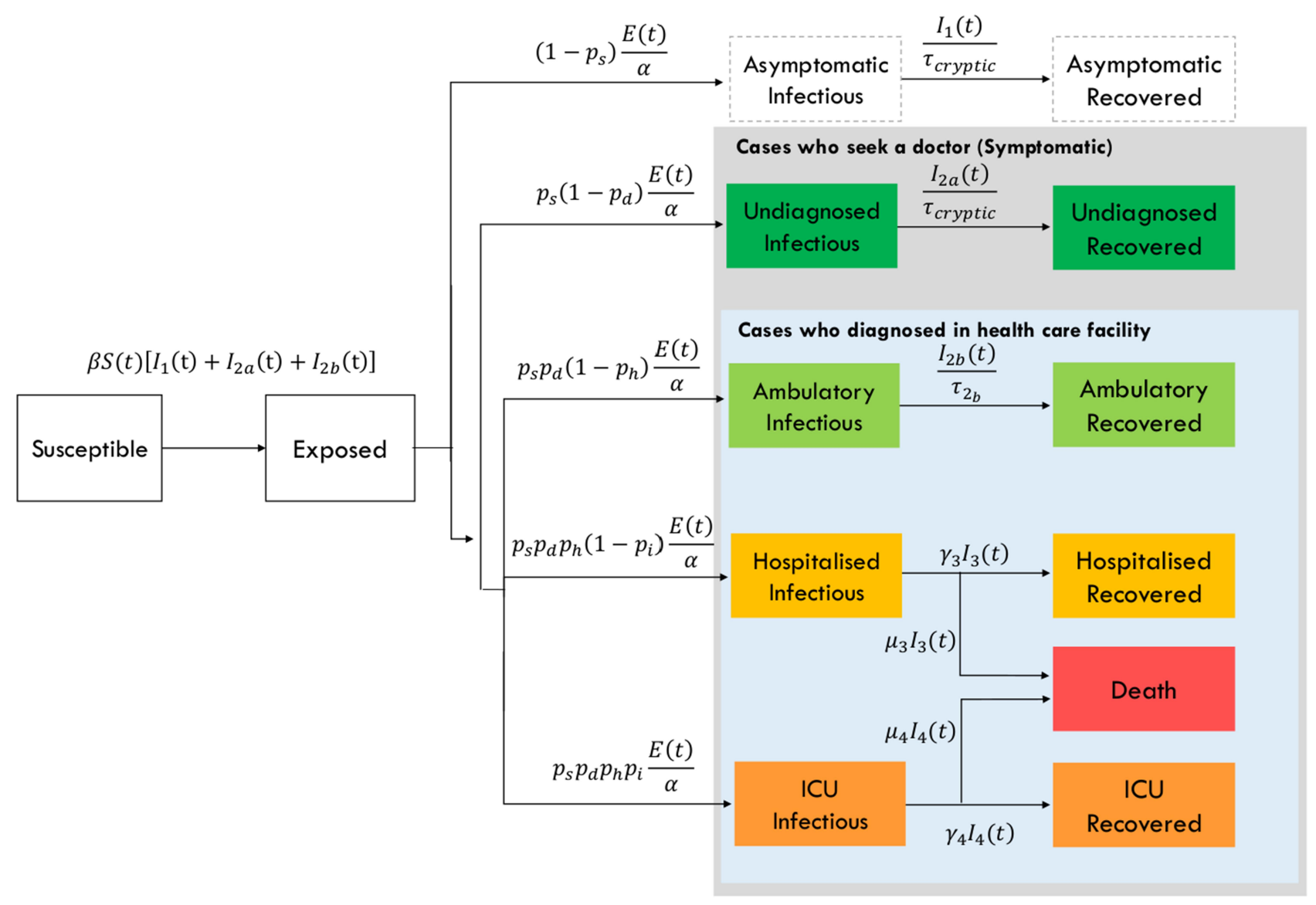

Figure I Flow chart of the model structure.

\section{Ratio of Confirmed and Suspected but Negative Cases}

Suspected cases are divided into negative cases and positive cases based on two consecutive coronavirus RT-PCR tests. Negative cases included those with mild symptoms that remain undiagnosed at healthcare facilities (level 2a) and suspected but negative cases. The positive cases included mild symptomatic ambulatory cases (level $2 b$ ), those requiring hospitalisation (level 3) and ICU cases (level 4). All suspected cases, who were quarantined in the isolation wards, were assumed to wait for three days in the isolation ward until laboratory confirmation. After three days, mild symptomatic ambulatory cases (level $2 \mathrm{~b}$ ) and mild symptoms that remain undiagnosed (level 2a) are discharged from the isolation ward.

To assess the effects of case misclassification, six ratios were used to reflect the proportion of suspected but negative cases with positive cases, ranging from 0 to $10(0,0.5,1,2,5$, 10). A zero ratio (1:0) has no case misclassification and conversely a ratio of 10 indicates significant uncertainty in preliminary diagnosis upon admission where a substantial proportion are incorrectly identified as being COVID-19 positive but later are confirmed to be negative. A separate set of analyses were conducted where rapid testing was implemented, or a zero ratio, at 2 to 5 months from the epidemic start point, to estimate its potential effects on usage of N95s.

\section{Results \\ Epidemiological Features in Simulated Outbreaks Under Scenarios}

A total of 4.5 million infections $(80 \%$ of the total population) were projected to occur in the severe scenario and 2.6 million $(45.6 \%)$ in the moderate or mild scenario over the span of a year. Hospitalized cases reached a peak at approximately 6.5 months (day 194), and 11 months (day 324) after the first case. At the peak, 65,000 hospitalised cases were estimated to occur in the severe epidemic, 15,000 in the moderate and 5000 in the mild scenario (Figure 2A). The peak in ICU admission occurred 3-4 weeks later than hospital admission (day 197 and day 328). At this time, the number of patients staying in the ICU amounted to 57,000, 9000 and 2000 (Figure 2B). 


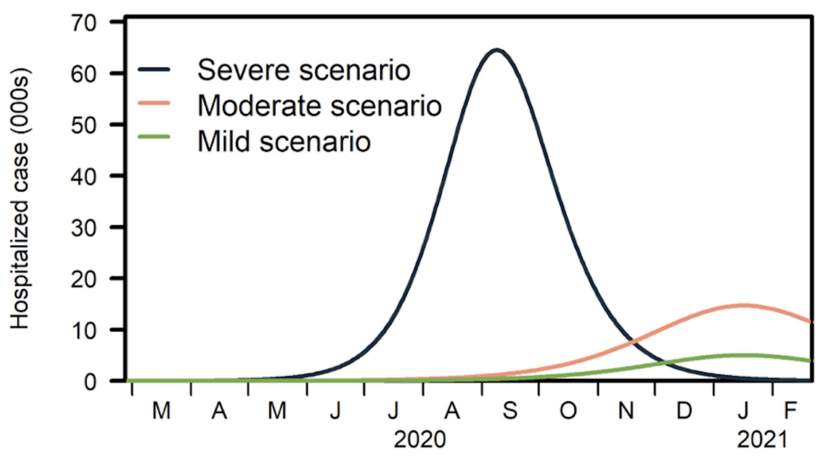

A

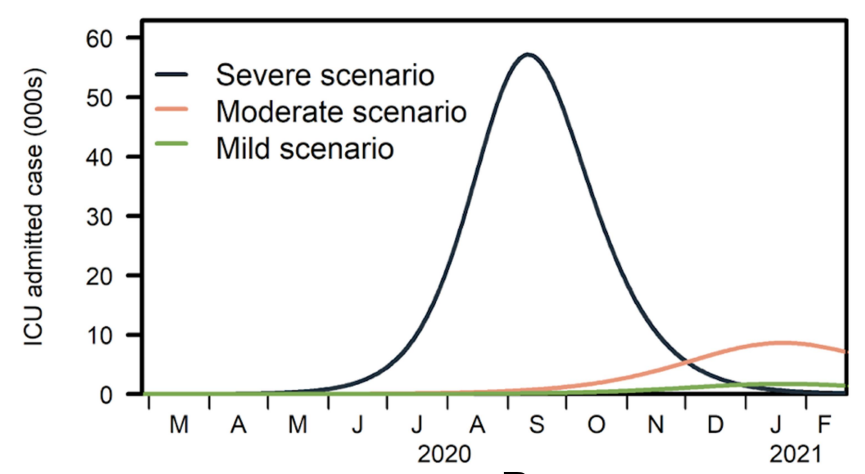

B

Figure 2 The epidemic curves in different scenarios (A) Hospitalized cases (B) ICU admitted cases. The colours indicate the scenarios. Black: Severe scenario, orange: moderate scenario, and green: mild scenario.

Final N95 Respirators Usage Comparison According to Different Scenarios

HCWs working in an ICU will use 72, 39, or 6 N95s per case per day in the single-use, extended-use and prolonged-use policies, respectively. Workers in the respiratory isolation ward will use 29,20 , or 4 per case per day in these respective policies. Full details are presented in the $\underline{\text { Supplementary Table } 2}$ and $\underline{3}$.

In the moderate scenario with the ratio of confirmed cases versus suspected cases at 1:0, the number of required N95s was found to be 117.1 million, 71.6 million and 12.8 million under the three policies. A reduction of $38.9 \%$ and $89.1 \%$ was observed in the final consumption when an extended-use and prolonged-use policy was implemented (Figure 3D and Supplementary Table 4). In the severe scenario, the final consumption amounted to 512.8 million, 304 million, and 52.4 million for each policy. The implementation of an extended or prolongeduse policy created further savings of $40.7 \%$ and $89.8 \%$ (Figure 3A and Supplementary Table 4). In the mild scenario, a total of 34.3 million, 21.9 million and 4.1 million N95s were estimated to be required for three policies. The reduction with extended and prolonged-use was $36.2 \%$ and $88.1 \%$ compared to the single-use policy (Figure $3 \mathrm{G}$ and Supplementary Table 4).

Under the mild scenario, the peak month of N95 consumption occurs 11 months later with the consumption of 10.3 million sets during the peak month according to the single-use policy, with a reduction of $35.9 \%$ for extendeduse and $87.4 \%$ in the prolonged-use policy (Supplementary Table 5). The peak month occurs 7 months later than in the severe scenario (Supplementary Table 5) where N95 consumption reached 188.1 million.
Compared to the single-use policy, the extended-use policy reduces N95 consumption by $40.6 \%$ and the prolonged-use policy by $89.7 \%$ (Supplementary Table 5 ).

We found that N95 consumption was sensitive to different ratios of confirmed cases versus suspected cases across the epidemic scenarios. With a ratio of $1: 2$, 313.4 million, 207 and 39.9 million N95s were estimated for use in each policy within the moderate scenario. The consumption increased by $\sim 3$ times in the severe epidemic scenario and reduced by $\sim 0.5$ in the mild scenario (Figure 3B, E and $\mathrm{H}$ ). With a high misclassification rate from a ratio of 1:10, N95 consumption amounted to 1.10 billion, 748.4 million, and 148.2 million in the moderate scenario across the three policies. This was substantially reduced by $31.8 \%$ and $86.5 \%$ within the extended and prolonged-use policies (Figure 3F and Supplementary Table 4).

In the severe scenario, 2.84 billion, 1.98 billion, and 386 million N95s were estimated to be used, representing a $30.4 \%$ and $86.4 \%$ reduction in the extended and prolongeduse policies (Figure 3C and Supplementary Table 4). In the mild scenario, N95 consumption was estimated to be 623.2 million, 428 million and 85.3 million, reducing final N95 consumption by $31.3 \%$ and $86.3 \%$ in the extended and prolonged-use policies (Figure 3I and Supplementary Table 4).

The peak month of N95 use in the moderate scenario was at 11 months where N95 demand was at 317.5 million according to single-use, 216.3 million for extended-use and 42.8 million for prolonged-use. However, for the severe scenario in the peak month occurring at seven months, N95 consumption increased to a high of 1.14 billion, 767.6 million and 

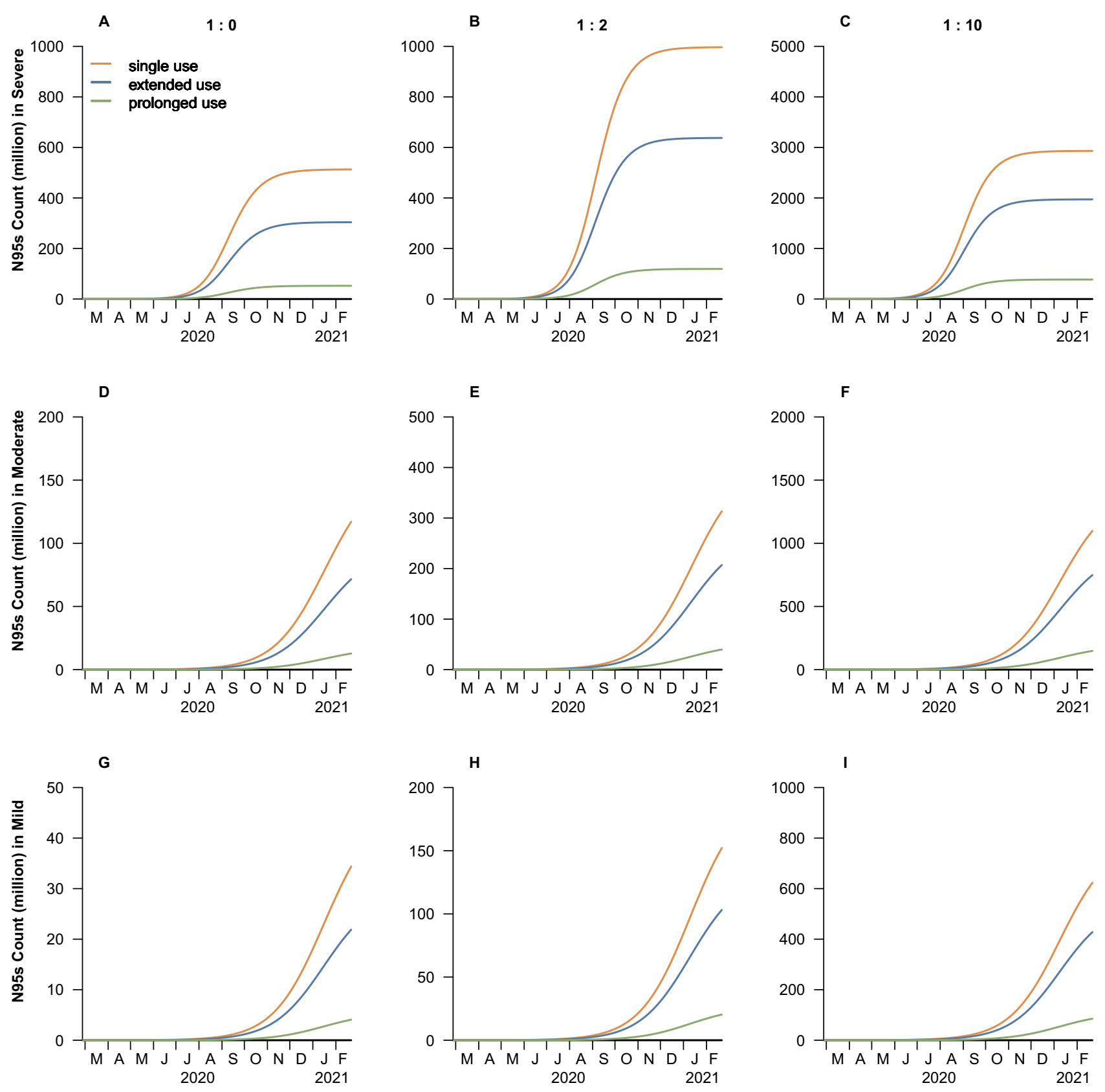

Figure 3 The cumulative N95s consumption for selected ratios of confirmed cases versus suspected cases. The simulated scenarios in plots (A-C) are severe, (D-F) moderate, and (G-I) mild. The ratios of confirmed cases versus suspected cases for: $(\mathbf{A}, \mathbf{D}$ and $\mathbf{G})$ is I:0; (B, E and $\mathbf{F})$ is I:2; (C, $\mathbf{F}$ and $\mathbf{I})$ is I:I0. The line colours describe policies of N95 usage where the red line shows single use, blue line shows extended use, and green line shows prolonged use.

150.7 million with these policies (Supplementary Table 5).

\section{N95 Respirators Usage with Implementation of Rapid Testing}

We also considered N95 usage in three epidemic scenarios under different policies with the introduction of rapid testing kits. The introduction of rapid diagnostic kits can reduce
N95 demand but are still required. In the severe scenario, a 5-month delay in switching to the the use of rapid diagnostic testing results in the usage of 620.6 million N95s with single-use, 413.1 million with extended-use and 80.1 million with prolonged-use policies. In the same epidemic scenario, if the rapid diagnostic is released within 2 months of the outbreak, N95 usage reduces substantially to 348.1 million, 225.1 million and 42.5 million, respectively 
(Figure 4A). In the mild and moderate epidemic scenario, the peaks for N95 use are reduced and delayed (Figure 3 and Supplementary Figure 4). In the moderate scenario, after a 5-month delay in switching to rapid diagnostic testing, 90.7 million, 60.8 million and 11.9 million N95s are required. A further reduction is observed if introduced at 2 months with 83.0 million, 55.4 million and 10.8 million N95s in demand (Figure 4B). Under the mild epidemic scenario, a 5-month and 2-month delay in the introduction of rapid diagnostic testing results in the N95 usage of 33.3 million and 28.6 million for single-use, 22.4 million and 19.2 million for extended-use, and 4.4 million and 3.8 million for prolonged-use (Figure 4C).

\section{Discussion}

Optimal N95 use is required for national and global supplies to be sustained, ensuring HCW safety and allowing for proper patient care across all healthcare facilities. We projected N95 usage across a wide range of scenarios where for a moderate epidemic scenario, our projections of consumption for a single-use policy with no misclassification of disease at hospital entry (ratio 1:0) was found to be 117.1 million, 71.6 million and 12.8 million. This reduced by $38.9 \%$ and $89.1 \%$ when extended and prolonged-use policies were implemented. These considerable reductions can extend the lifetime of N95 stocks across time and allow for a wider distribution between different regions or countries to reduce the stress on demand. When more case misclassification occurrs at a ratio of 1:10, N95 use increased to 1.10 billion, 748.4 million, and 148.2 million, which would be considerably more challenging to obtain and distribute effectively. The introduction of a rapid diagnostic kit at any point of the epidemic which can immediately ascertain if an individual is SARS-CoV-2 positive therefore advantageous and should be immediately implemented when possible provided test accuracy is high.

Our approach can be utilised elsewhere with considerations of the implementation of such extended-use policies. This is especially paramount at this time as the WHO, despite already shipping more than 900,000 surgical masks, 62,000 N95s and 34,000 face shields with other PPEs to 133 countries to supplement supplies for those at contingency or crisis capacities, has stated that overall global supplies are rapidly depleting. ${ }^{3}$ This is further complicated by the fact that the PPE supply chain landscape is complex. Numerous national PPE stockpiling systems exist, such as the US Strategic National Stockpile, Canada's National Emergency Strategic Stockpile, Australia's National Medical Stockpile and Taiwan's 3-tier stockpile. ${ }^{19}$ These often require multiple private contractors and vendors to balance procurement and deployment to facilitate demand, and require prioritization in allocating resources to different hospitals and healthcare
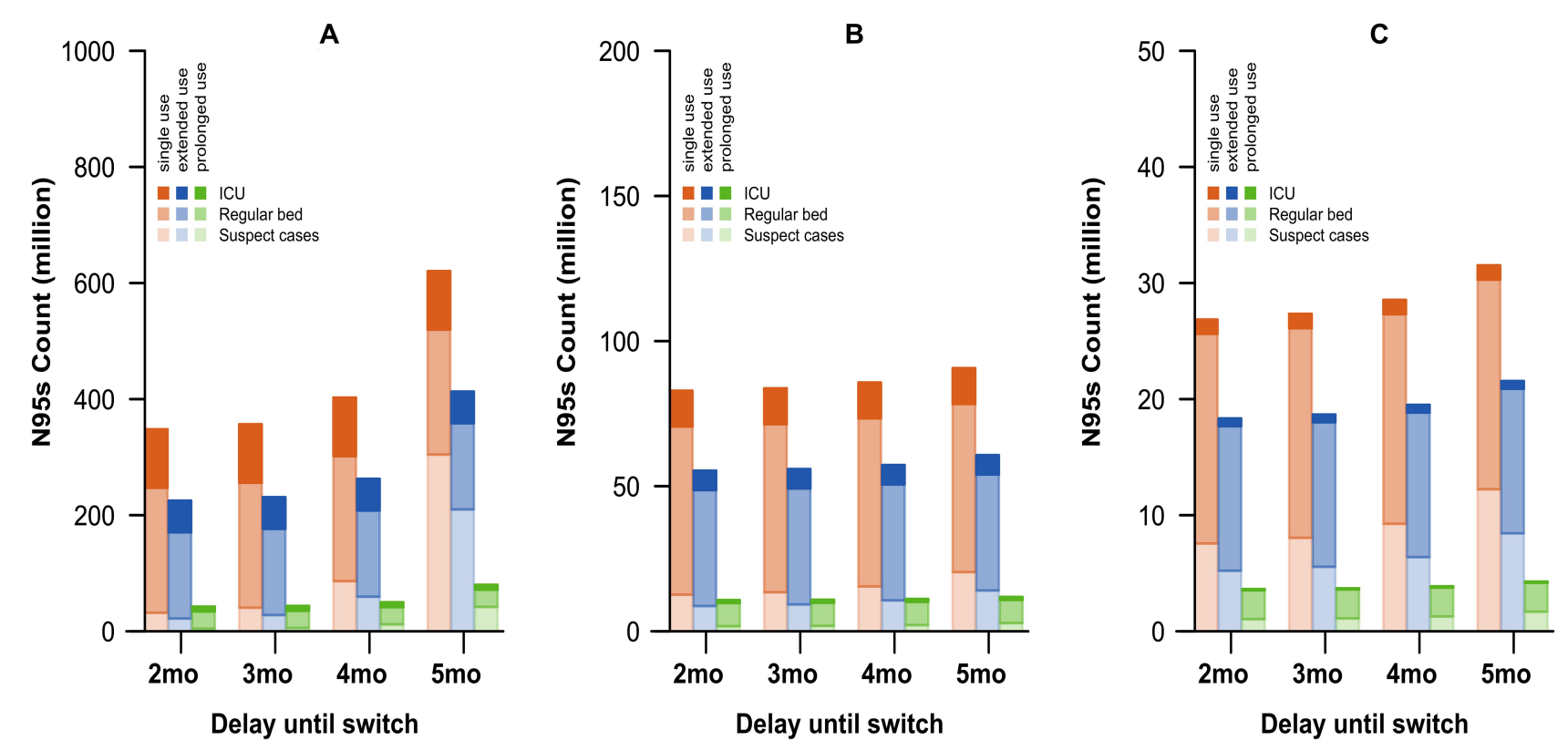

Figure 4 N95 use across time with the switch to rapid testing in different epidemic scenarios where $(\mathbf{A})$ is severe, $(\mathbf{B})$ is moderate and $(\mathbf{C})$ is mild. The orange line series shows single use, blue the extended-use and green the prolonged-use policies. Each bar is stratified by the levels of health care setting. Darker colours describe ICU cases, medium shading the cases in regular beds and lighter shades the suspected COVID-19 cases. 
centres with the ongoing supply strain. Manufacturers, particularly in China, are also struggling to ramp up production due to the travel restrictions and quarantining procedures in place which requires other strategies to be considered such as extended-use.

During the last pandemic (influenza A(H1N1-2009)), Hashikura and Kizu recommended a minimum of 8 weeks of PPE supply for stockpiling with 4 sets being utilised per day by high-risk workers, 2 sets for medium and low-risk groups, 2 surgical masks for each worker and inpatient, and 1 for every suspected case. ${ }^{20}$ Recommendations at the Ministry of Health, Singapore, are more risk averse by aiming to maintain a 3- to 6-month stockpile, with individual capacities set for each medical institution. ${ }^{21}$ The overall stockpile capacity is a function of the renewal rate among workers, the protective robustness of the equipment and corresponding costs. Based on our findings, the extended-use policy could be utilised within wards before supply chains are stressed where multiple infected patients are being visited simultaneously, provided infection risk can be mitigated. Should infection become widespread with significant patient load at healthcare facilities, prolonged-use may have to be utilised to prevent supply shortages. Where possible, it is recommended that gowns and N95 use be prioritized for high-risk aerosol-generating procedures including endotracheal intubation and bronchoscopy. ${ }^{11}$ With $42 \%$ of 52 ICU patients requiring invasive mechanical ventilation and $13.5 \%$ of this group acquiring the infection in hospital, considerable transmission risk exists. ${ }^{22,23}$ This risk is further exacerbated by the presence of environmental contamination from patients. ${ }^{24}$

Singapore is currently at an orange level of its Disease Outbreak Response System Condition, which reflects the high risk of case importation and ongoing outbreaks within foreign worker dormitories. Although PPE, including N95s, only forms a component of protective procedures, it remains crucial alongside limiting the number of encounters, implementation of negative pressure isolation rooms, physical barriers and exclusion of non-COVID-19 patients by testing. Communication with the public remains the first barrier of defence with recommendations of appropriate mask usage and prioritization of supplies for HCWs. Public engagement will not be sufficient however as infection events will continue to occur; therefore, countries facing even greater short and long-term shortages of N95s could potentially consider the policy findings here for implementation. ${ }^{25}$

As with all modelling studies, many assumptions were made. Firstly, the SEIR model did not account for any heterogeneities in the infection or symptomatic rates as a result of factors such as the age structure. We also did not consider the effects of ongoing control methods, primarily social distancing, on the number of infections through time. This is due to Singapore's outbreak in the community being kept relatively small, making estimations of the control's effects very challenging. Secondly, parameters such as the ratio of confirmed and suspected cases remain largely unknown. Thirdly, the number of N95s used assumes all healthcare workers adhere to the guidelines, not counting for accidents or personal preferences, which may inflate the requirements. It is furthermore unknown as to whether the reuse will cause an increased risk of infection among HCWs due to mask degradation. Fourthly, hospital procedures may change where fewer workers stay longer in N95s within the respiratory isolation wards so as to avert N95 use. Lastly, the proportions allocated to each level here may change according to specifications set out by policymakers should resources become more limited.

Determined by the epidemic size, an extended-use policy should be considered where short-term supply chains are strained but planning measures are in place to ensure long-term availability. With severe shortage expectations from a severe epidemic, prolonged-use can be proposed as a necessary policy to significantly prolong supply. Should infection become widespread with significant patient load at healthcare facilities, extensive reuse, via the extended-use and prolonged-use policy, may also have to be utilised to prevent supply shortages.

\section{Abbreviations}

PPE, personal protective equipment; HCW, healthcare worker; WHO, World Health Organisation; SEIR model, Susceptible-Exposed-Infectious-Recovered model; ICU, intensive care unit.

\section{Acknowledgments}

This research is supported by the Singapore's National Medical Research Council under the Centre Grant Programme - Singapore Population Health Improvement Centre (NMRC/CG/C026/2017_NUHS) and COVID-19 Research Fund (COVID19RF-004); and by the JSPS KAKENHI Grant Number 19K24250, and Leading Young Researcher Overseas Visit Program under Ministry of Education, Culture, Sports, Science and Technology, Japan. 


\section{Disclosure}

The authors declare that they have no competing interests. These authors contributed equally to this manuscript: Hirono Otomaru and Borame L Dickens.

\section{References}

1. Koplan JP, Butler-Jones D, Tsang T, Yu W. Public health lessons from severe acute respiratory syndrome a decade later. Emerg Infect Dis. 2013;19(6):861-863. doi:10.3201/eid1906.121426

2. Lee SI. Costly lessons from the 2015 Middle East Respiratory Syndrome coronavirus outbreak in Korea. J Prev Med Public Health. 2015;48(6):274-276. doi:10.3961/jpmph.15.064

3. World Health Organization. Rolling updates on coronavirus disease (COVID-19). Available from: https://www.who.int/emergencies/dis eases/novel-coronavirus-2019/events-as-they-happen. Accessed August 03, 2020.

4. Ranney ML, Griffeth V, Jha AK. Critical supply shortages - the need for ventilators and personal protective equipment during the Covid-19 pandemic. N Engl J Med. 2020;382(18):e41. doi:10.1056/ NEJMp2006141

5. World Health Organization. Shortage of personal protective equipment endangering health workers worldwide. Available from: https:// www.who.int/news-room/detail/03-03-2020-shortage-of-personalprotective-equipment-endangering-health-workers-worldwide. Accessed April 21, 2020.

6. COVID-19: how much protection do face masks offer? Available from: https://www.mayoclinic.org/diseases-conditions/coronavirus/in-depth/cor onavirus-mask/art-20485449. Accessed September 08, 2020.

7. Zhang M, Emery AR, Tannyhill RJ, Zheng H, Wang J. Surgical masks or N95 respirators for OMF surgery during COVID-19 pandemic. J Oral Maxillofac Surg. 2020. doi:10.1016/j.joms.2020.08.024

8. Centers for Disease Control and Prevention. Interim infection prevention and control recommendations for patients with confirmed coronavirus disease 2019 (COVID-19) or persons under investigation for COVID-19 in healthcare settings; 2020; Available from: https:// www.cdc.gov/coronavirus/2019-ncov/hcp/infection-control.html. Accessed October 9, 2020.

9. World Health Organization. Rational use of personal protective equipment for coronavirus disease (COVID-19): interim guidance, 27 February 2020. Available from: https://apps.who.int/iris/handle/ 10665/331215. Accessed August 03, 2020.

10. Beckman S, Materna B, Goldmacher S, et al. Evaluation of respiratory protection programs and practices in California hospitals during the 2009-2010 H1N1 influenza pandemic. Am J Infect Control. 2013;41(11):1024-1031. doi:10.1016/j.ajic.2013.05.006

11. Centers for Disease Control and Prevention. Recommended guidance for extended use and limited reuse of N95 filtering facepiece respirators in healthcare settings. Available from: https:/www.cdc.gov/ niosh/topics/hcwcontrols/recommendedguidanceextuse.html. Accessed August 03, 2020.
12. Kobayashi LM, Marins BR, Costa P, Perazzo H, Castro R. Extended use or reuse of N95 respirators during COVID-19 pandemic: an overview of national regulatory authority recommendations. Infect Control Hosp Epidemiol. 2020;1-3.

13. Rodriguez-Martinez CE, Sossa-Briceno MP, Cortes JA. Decontamination and reuse of N95 filtering facemask respirators: a systematic review of the literature. Am J Infect Control. 2020. doi:10.1016/j.ajic.2020.07.004

14. Guan WJ, Ni ZY, Hu Y, et al. Clinical characteristics of coronavirus disease 2019 in China. N Engl J Med. 2020;382(18):1708-1720. doi:10.1056/NEJMoa2002032

15. Wang D, Hu B, Hu C, et al. Clinical characteristics of 138 hospitalized patients with 2019 novel coronavirus-infected pneumonia in Wuhan, China. JAMA. 2020.

16. World Health Organization. Coronavirus disease 2019 (COVID-19) situation report-191. Available from: https://www.who.int/docs/ default-source/coronaviruse/situation-reports/20200729-covid-19sitrep-191.pdf?sfvrsn=2c327e9e_2. Accessed August 03, 2020.

17. Mizumoto K, Chowell G. Estimating risk for death from 2019 novel coronavirus disease, China, January-February 2020. Emerg Infect Dis. 2020;26(6):1251-1256. doi:10.3201/eid2606.200233

18. Park M, Cook AR, Lim JT, Sun Y. A systematic review of COVID-19 epidemiology based on current evidence. J Clin Med. 2020.

19. Chen YJ, Chiang PJ, Cheng YH, et al. Stockpile model of personal protective equipment in Taiwan. Health Secur. 2017;15(2):170-174. doi:10.1089/hs.2016.0103

20. Hashikura M, Kizu J. Stockpile of personal protective equipment in hospital settings: preparedness for influenza pandemics. Am J Infect Control. 2009;37(9):703-707. doi:10.1016/j.ajic.2009.05.002

21. Ministry of Health Singapore. Moh pandemic readiness and response plan for influenza and other acute respiratory diseases (Revised April 2014). Available from: https://www.moh.gov.sg/docs/librariesprovi der5/diseases-updates/interim-pandemic-plan-public-ver-_april-2014. pdf. Accessed August 03, 2020.

22. Yang X, Yu Y, Xu J, et al. Clinical course and outcomes of critically ill patients with SARS-CoV-2 pneumonia in Wuhan, China: a single-centered, retrospective, observational study. Lancet Respir Med. 2020;8(5):475-481. doi:10.1016/S2213-2600(20)30079-5

23. Cheung JC, Ho LT, Cheng JV, Cham EYK, Lam KN. Staff safety during emergency airway management for COVID-19 in Hong Kong. Lancet Respir Med. 2020;8(4):e19. doi:10.1016/S2213-2600(20) 30084-9

24. Ong SWX, Tan YK, Chia PY, et al. Air, surface environmental, and personal protective equipment contamination by severe acute respiratory syndrome coronavirus 2 (SARS-CoV-2) from a symptomatic patient. JAMA. 2020;323(16):1610. doi:10.1001/ jama.2020.3227

25. Dong E, Du H, Gardner L. An interactive web-based dashboard to track COVID-19 in real time. Lancet Infect Dis. 2020;20(5):533-534. doi:10.1016/S1473-3099(20)30120-1
Risk Management and Healthcare Policy

\section{Publish your work in this journal}

Risk Management and Healthcare Policy is an international, peerreviewed, open access journal focusing on all aspects of public health, policy, and preventative measures to promote good health and improve morbidity and mortality in the population. The journal welcomes submitted papers covering original research, basic science, clinical \& epidemiological studies, reviews and evaluations, guidelines, expert opinion and commentary, case reports and extended reports. The manuscript management system is completely online and includes a very quick and fair peer-review system, which is all easy to use. Visit http://www.dovepress.com/testimonials.php to read real quotes from published authors. 